\title{
RELACIÓN FENOLOGÍA-CLIMA DE CUATRO ESPECIES DE ORQUÍDEAS EN UN BOSQUE ALTOANDINO DE COLOMBIA
}

\author{
Juan Camilo Ordóñez-Blanco ${ }^{1,2} \&$ Ángela Parrado-Rosselli ${ }^{1}$ \\ ${ }^{1}$ Maestría en Manejo Uso y Conservación del Bosque, Universidad Distrital Francisco José de Caldas, \\ Calle 72 no 88-44 Apto 103, Código Postal 111711, Bogotá, Bogotá D. C., Colombia \\ ${ }^{2}$ Autor para correspondencia: phaleno@gmail.com
}

\begin{abstract}
AвSTRACT. The objective of this research was to characterize, during an annual cycle, the relationship between weather and the phenology of four common orchid species in a high-Andean forest of the Eastern Cordillera of Colombia. Flowering and fruiting of 30 adult individuals of Cyrtochilum revolutum, Malaxis excavata, Ponthieva diptera and Stelis pulchella were monitored on a monthly basis. The number of flowering buds, open flowers, unripe and ripe fruits (closed and open capsule, respectively) were correlated to climatic variables (i.e. mean monthly precipitation, mean, minimum and maximum monthly temperature and, relative humidity) obtained during the same time period. The results showed that all orchid species exhibited reproductive activity during the studied year. C. revolutum only flowered, and was positively correlated with rainfall. The flowering peak of $S$. pulchella, occurred during the coldest months when relative humidity was also low. In contrast, the peak of unripe fruits was recorded when both the relative humidity and mean temperature were the highest, while capsules opened during the driest months. Flowering and fruiting of M. excavata were extended, lasting more than four months. The greatest number of open flowers was positively correlated to precipitation, while the number of unripe fruits was correlated with relative humidity and rainfall of twomonths prior. Few individuals of $P$. diptera flowered and fruited during the studied year, while reproductive activity was correlated to rainfall and temperature. Although the relationship between climatic variables and phenology is differential between species, our findings consistently showed that three out of four of the studied species (C. revolutum, $M$. excavata y $P$. diptera) flowered during the rainy season with significant positive correlations, which is discussed in light of literature findings.
\end{abstract}

RESUMEN. El objetivo de esta investigación fue caracterizar, durante un ciclo anual, la relación fenología-clima de cuatro especies comunes de orquídeas en un bosque altoandino de la Cordillera Oriental colombiana. Se monitoreó mensualmente la floración y la fructificación de 30 individuos adultos de Cyrtochilum revolutum, Malaxis excavata, Ponthieva diptera y Stelis pulchella. El número de flores en botón, flores abiertas, frutos inmaduros (cápsula cerrada) y el número de frutos maduros (cápsula abierta) se correlacionaron con las variables climáticas precipitación media mensual, temperatura media, mínima y máxima mensuales y humedad relativa mensual del mismo período de estudio. Los resultados mostraron que las cuatro especies de orquídeas presentaron actividad reproductiva durante el año de seguimiento. C. revolutum sólo floreció y se correlacionó de forma positiva con la precipitación. El pico de floración de S. pulchella, ocurrió en los meses más fríos y con menor humedad relativa. En contraste, la producción de frutos verdes se presentó en los momentos de mayor humedad y mayor temperatura, mientras que la apertura de las cápsulas ocurrió en los meses más secos. M. excavata presentó un patrón de floración y de fructificación extendido y el mayor número de flores abiertas se correlacionó de forma positiva con la precipitación. La producción de frutos verdes se correlacionó con la humedad relativa y la precipitación de dos meses atrás. Aunque $P$. diptera mostró pocos individuos en flor y en fruto, la actividad reproductiva se correlacionó con la precipitación y con la temperatura. A pesar de que la relación entre las variables climáticas y la fenología es diferente entre las especies, para tres de las cuatro especies estudiadas (C. revolutum, M. excavata y P. diptera) fue consistente la ocurrencia de la mayor floración durante la época de lluvias con correlaciones positivas significativas, aspecto que se discute a la luz de estudios previos.

KEY wORDS: Andean forest, climatic variables, flowering, fruiting, Orchidaceae 
Introducción. Las orquídeas son un grupo de plantas de gran importancia en términos ecológicos y económicos. Estas son atractivas en términos del ecoturismo, el biocomercio y la bioprospección (Cozzolino \& Widmer 2005, Giraldo \& Betancur 2011, Ballantyne \& Pickering 2012, Parra 2013); así como también han sido consideradas como indicadores del estado de salud de los ecosistemas (Gentry \& Dodson 1987, Pineda 2004). Esto ha generado que hayan sido estudiadas desde diversos campos que involucran inventarios, ecología e incluso tecnologías en métodos de propagación y cultivo ex situ (Schweinfurth 1958, Dunsterville \& Garay 1979, Ackerman 1992, Escobar 1994, Romero \& Carnevali 2000, Dodson 2003, Hágsater \& Soto 2008, Otero \& Bayman 2009, Giraldo \& Betancur 2011).

El cultivo de las orquídeas bajo condiciones ex situ se ha intensificado en los últimos años y constituye un renglón promisorio en el sector de la floricultura y la restauración ecológica (Ballantyne \& Pickering 2012). Sin embargo, se han identificado limitantes considerables en la adaptación y reproducción, puesto que sólo algunas especies o individuos logran reproducirse bajo condiciones controladas (Singer 2009, Quiroga et al. 2010). En el caso de las orquídeas altoandinas, observaciones personales muestran que muchas especies presentan pobre desarrollo, mortalidad y baja reproducción en condiciones ex situ, lo que se debe, en gran medida, a que se desconocen las condiciones climáticas que se deben procurar con el fin de lograr su aclimatación y manejo. Por lo tanto, el conocimiento del efecto de las variables ambientales sobre los eventos de floración y fructificación de las especies podría ayudar a entender las condiciones que deben ser manejadas a la hora de aplicar estrategias de manejo reproductivo ex situ.

La fenología de floración y fructificación de las especies de plantas tropicales ha mostrado una gran relación con factores tanto bióticos como abióticos (van Schaik et al. 1993). Para el caso de las orquídeas, los patrones de floración han sido atribuidos principalmente a la precipitación, en donde el aumento de las lluvias se relaciona con el inicio de la floración (Ibarra et al. 1991, Reich 1995, Lemus-Jiménez \& Ramírez 2002). Respecto a la fructificación, esta se ha asociado comúnmente a las temporadas secas dado el carácter anemocórico de la mayoría de las especies (Diez 2002, Roldán \& Larrea 2003, Vieira-Faria et al. 2007).
Así, el objetivo de esta investigación fue caracterizar, durante un ciclo anual, la relación fenología-clima de cuatro especies comunes de orquídeas en un relicto de bosque altoandino de la Cordillera Oriental colombiana. Para tal fin, se caracterizó durante un año la fenología reproductiva (floración y fructificación) de cuatro especies de orquídeas (Cyrtochilum revolutum, Stelis pulchella, Malaxis excavata y Ponthieva diptera) y se correlacionó con las variables climáticas: temperatura media, mínima y máxima, humedad relativa y precipitación media durante el período de estudio. A pesar de que en la actualidad, los estudios fenológicos multianuales proveen información más robusta sobre las dinámicas reproductivas de las especies (Hudson \& Keatley 2009), dado el escaso conocimiento sobre la fenología de las orquídeas colombianas, consideramos que este estudio de un año, constituye un importante aporte para el mejor entendimiento de la ecología de las orquídeas tropicales.

Materiales y métodos. Esta investigación se desarrolló en un relicto de bosque altoandino de la Cordillera Oriental, ubicado en el municipio de La Calera, Cundinamarca, Colombia $\left(4^{\circ} 43^{\prime} 6.8^{\prime \prime} \mathrm{N}\right.$ $\left.73^{\circ} 58^{\prime} 9.26^{\prime \prime} \mathrm{W}\right)$. El área de estudio se encuentra en un rango altitudinal entre los 2815-3050 m. La zona presenta una temperatura media anual entre los 14 y $16^{\circ} \mathrm{C}$ y un régimen bimodal de lluvias, con una precipitación media anual de $970 \mathrm{~mm}$, siendo mayo y noviembre las épocas más húmedas (Caballero et al. 1997, Montenegro \& Vargas 2005, Guerrero et al. 2008). Por estar situada en la zona ecuatorial las horas luz son relativamente constantes a lo largo del año y no difieren más de 30 minutos (Borchert et al. 2015).

La vegetación predominante corresponde a relictos de bosque secundario, donde las familias de árboles más abundantes son Melastomataceae y Winteraceae (Vargas 1986, Caballero et al. 1997, Montenegro \& Vargas 2005). Además, los géneros más comunes son: Clethra (Clethraceae), Clusia (Clusiaceae), Drimys (Winteraceae), Hesperomeles (Rosaceae) y Weinmannia (Cunoniaceae; Vargas 1986, Caballero et al. 1997, Montenegro \& Vargas 2005).

Las orquídeas estudiadas corresponden a cuatro especies altoandinas silvestres de amplia distribución y con abundante número de individuos en la zona de estudio. Estas son Cyrtochilum revolutum (Lindl.) 
Dalström, Stelis pulchella Kunth., Malaxis excavata (Lindl.) Kuntze y Ponthieva diptera Linden \& Rchb.f. y (Fig. 1). Estas especies son de variados hábitos y tipos de crecimiento, es así que $C$. revolutum se desarrolla como semiterrestre (Baker \& Baker, 2006); S. pulchella crece tanto como semiterrestre o litófita, mientras que M. excavata y $P$. diptera son terrestres pequeñas e inconspicuas (Tabla 1). Según la literatura de orquídeas emparentadas con las especies de estudio $C$. revolutum es polinizada por abejas y avispas (Torreta et al. 2011) y $S$. pulchella, $M$. excavata y $P$. diptera son polinizadas principalmente por moscas (entomofilia; Borba \& Semir 2001, Kite et al. 2008, Duque-Buitrago et al. 2014).

Toma de datos - Se monitoreó la floración y fructificación de las cuatro especies de forma mensual durante un ciclo anual entre agosto de 2013 y agosto de 2014. Los registros se tomaron para 30 individuos por especie en estado adulto. Con el fin de minimizar
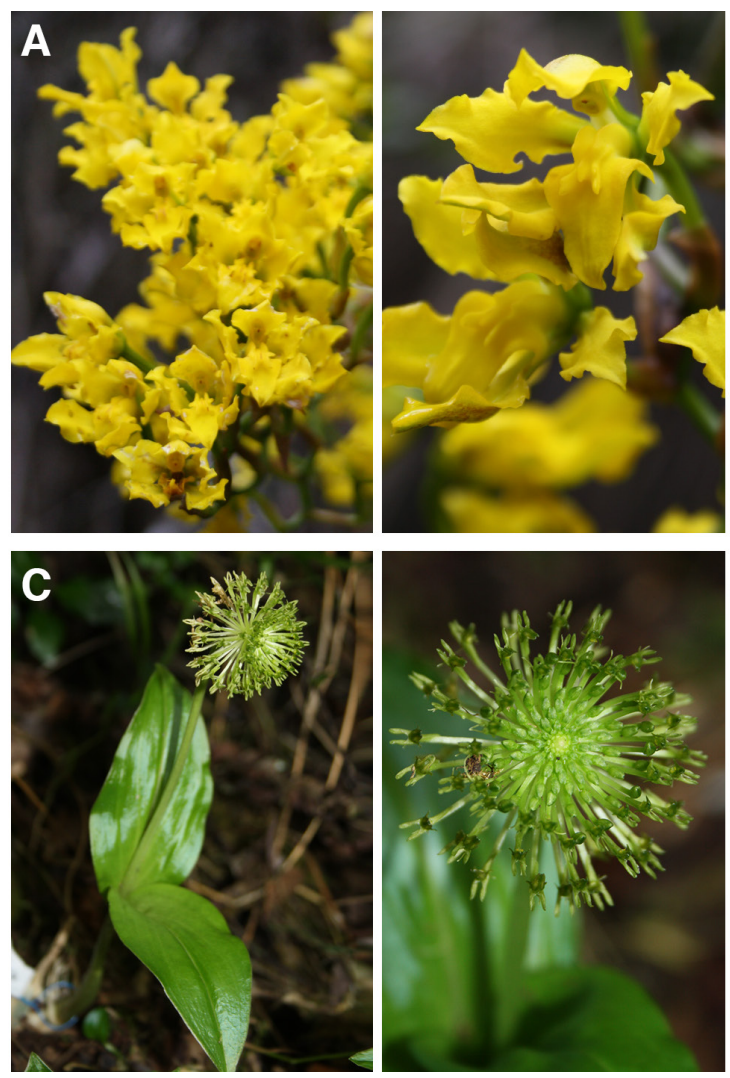

el efecto del microclima en la fenología de las especies la ubicación de los ejemplares se realizó al azar desde el suelo hasta la altura de $2 \mathrm{~m}$ sobre los forófitos (Parra 2013). A pesar de lo anterior, no se encontraron individuos por encima de los $50 \mathrm{~cm}$ del suelo, por lo que la variabilidad en términos de la distribución horizontal fue mínima. Adicionalmente, para los casos de especies terrestres y semiterrestres se muestrearon individuos agregados teniendo en cuenta que fueran distintos entre sí.

La floración de cada individuo se registró en términos del número de flores en botón, número de flores abiertas (Talora \& Morellato 2000, Roldán \& Larrea 2003, Tremblay et al. 2006, Ely-Bali et al. 2010, Torretta et al. 2011, Sánchez-Landaverde 2014). La fructificación se registró en términos del número de frutos inmaduros (cápsula cerrada) y del número de frutos maduros (cápsula abierta) con base en Talora \& Morellato (2000) y Roldán \& Larrea (2003).
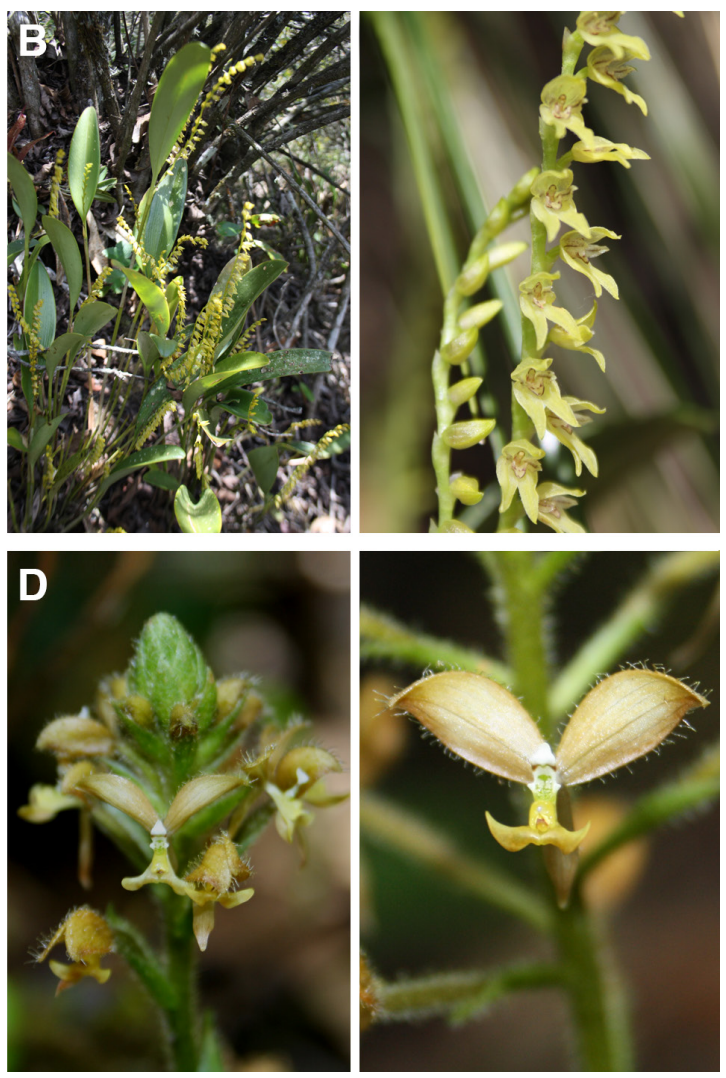

Figura 1. Especies estudiadas en un bosque altoandino de la Cordillera Oriental de Colombia. A. Cyrtochilum revolutum (Lindl.) Dalström, B. Stelis pulchella Kunth, C. Malaxis excavata (Lindl.) Kuntze y D. Ponthieva diptera Linden \& Rchb.f. 
TABLA 1. Características generales de las especies de estudio.

\begin{tabular}{|c|c|c|c|c|c|}
\hline Especie & Subfamilia & \multicolumn{2}{|l|}{ Tribu } & Subtribu & Hábito \\
\hline $\begin{array}{l}\text { Cyrtochilum revolutum } \\
\text { (Lindl.) Dalström }\end{array}$ & Epidendroideae & Cymbidieae & \multicolumn{2}{|c|}{ Oncidiinae } & Semiterrestre \\
\hline Stelis pulchella Kunth & Epidendroideae & \multicolumn{2}{|l|}{ Epidendreae } & Pleurothallidinae & Semiterrestre-Litófita \\
\hline $\begin{array}{l}\text { Malaxis excavata (Lindl.) } \\
\text { Kuntze }\end{array}$ & Epidendroideae & Malaxideae & \multicolumn{2}{|c|}{ Malaxidinae } & Terrestre \\
\hline $\begin{array}{l}\text { Ponthieva diptera Linden \& } \\
\text { Rchb.f. }\end{array}$ & Orchidoideae & Cranichideae & \multicolumn{2}{|c|}{ Cranichidinae } & Terrestre \\
\hline Especie & $\begin{array}{l}\text { Tipo de } \\
\text { crecimiento }\end{array}$ & $\begin{array}{l}\text { Estructuras } \\
\text { vegetativas }\end{array}$ & $\begin{array}{l}\text { Sindrome de } \\
\text { polinización }\end{array}$ & Taxa & Referencia \\
\hline $\begin{array}{l}\text { Cyrtochilum revolutum } \\
\text { (Lindl.) Dalström }\end{array}$ & Simpodial & Pseudobulbos & Entomofilia & Hymenoptera & Torreta et al. 2011 \\
\hline Stelis pulchella Kunth & Simpodial & $\begin{array}{l}\text { Ramicaules y hojas } \\
\text { coriáceas }\end{array}$ & Entomofilia & Díptera & $\begin{array}{l}\text { Duque-Buitrago et al. } \\
2014\end{array}$ \\
\hline $\begin{array}{l}\text { Malaxis excavata (Lindl.) } \\
\text { Kuntze }\end{array}$ & Simpodial & Cormos & Entomofilia & Díptera & Kite et al. 2008 \\
\hline $\begin{array}{l}\text { Ponthieva diptera Linden \& } \\
\text { Rchb.f. }\end{array}$ & Monopodial & $\begin{array}{l}\text { Hojas arrosetadas y } \\
\text { con tricomas }\end{array}$ & Entomofilia & Díptera & $\begin{array}{l}\text { Borba \& Semir 2001, } \\
\text { Kite et al. 2008, Duque- } \\
\text { Buitrago et al. } 2014\end{array}$ \\
\hline
\end{tabular}

Todos los individuos se marcaron y enumeraron utilizando etiquetas plásticas según su aparición en el campo. Se recolectaron ejemplares testigo, los cuales fueron procesados de acuerdo a las técnicas estándar de herborización y depositados en el Herbario UDBC. La identificación de estas especies se hizo a partir de la revisión de fuentes bibliográficas especializadas y consulta de los ejemplares que hacen parte de la colección en los herbarios JBB (Jardín Botánico de Bogotá José Celestino Mutis), UDBC (Universidad Distrital Francisco José de Caldas), HUA (Universidad de Antioquia) y COL (Nacional Colombiano). Adicionalmente y con el objeto de identificar patrones fenológicos de las especies a partir de muestras de herbario, se registró la fecha de la floración y la fructificación de los ejemplares fértiles. Sin embargo, debido al bajo número de ejemplares no fue posible obtener un patrón fenológico para cada una de las especies.

Los datos de precipitación se obtuvieron de la Empresa de Acueducto de Bogotá de la estación climatológica del Embalse San Rafael, localizada a 1.7 $\mathrm{km}$ del sitio de muestreo y a $2809 \mathrm{~m}$ de altura. Los datos de temperatura media, máxima y mínima, y humedad relativa media se obtuvieron de la estación de Guasca del IDEAM ubicada a $12.2 \mathrm{~km}$ del sitio y $2750 \mathrm{~m}$ de altura. Aunque esta estación se encuentra relativamente distante de la zona de estudio, corresponde al mismo flanco de la cordillera e igual rango altitudinal, por lo que se consideró que los valores de temperatura y humedad relativa son similares a los del área de estudio.

Análisis de datos —. La duración de la floración/ fructificación se estableció de acuerdo con lo sugerido por Newstrom et al. (1994) y Stevenson et al. (2008) como breve ( $<1$ mes), intermedia (1-5 meses) y extendida ( $>5$ meses). Si la especie no presentó patrones de duración detectables se consideró como irregular (Parada et al. 2012). También se clasificó como continua (floración/fructificación a lo largo del año por un período mayor a seis meses), episódica (producción de flores/frutos más de una vez por año en eventos discontinuos de menos de seis meses) y anual (únicamente un ciclo por año) de acuerdo con Newstrom et al. (1994) y Stevenson et al. (2008).

Para cada especie se analizaron las variaciones de los eventos fenológicos entre cada mes por medio de una ANOVA de medidas repetidas. Con el fin de establecer la relación entre la ocurrencia de las fenofases con las variables climáticas mensuales, se realizó un análisis 

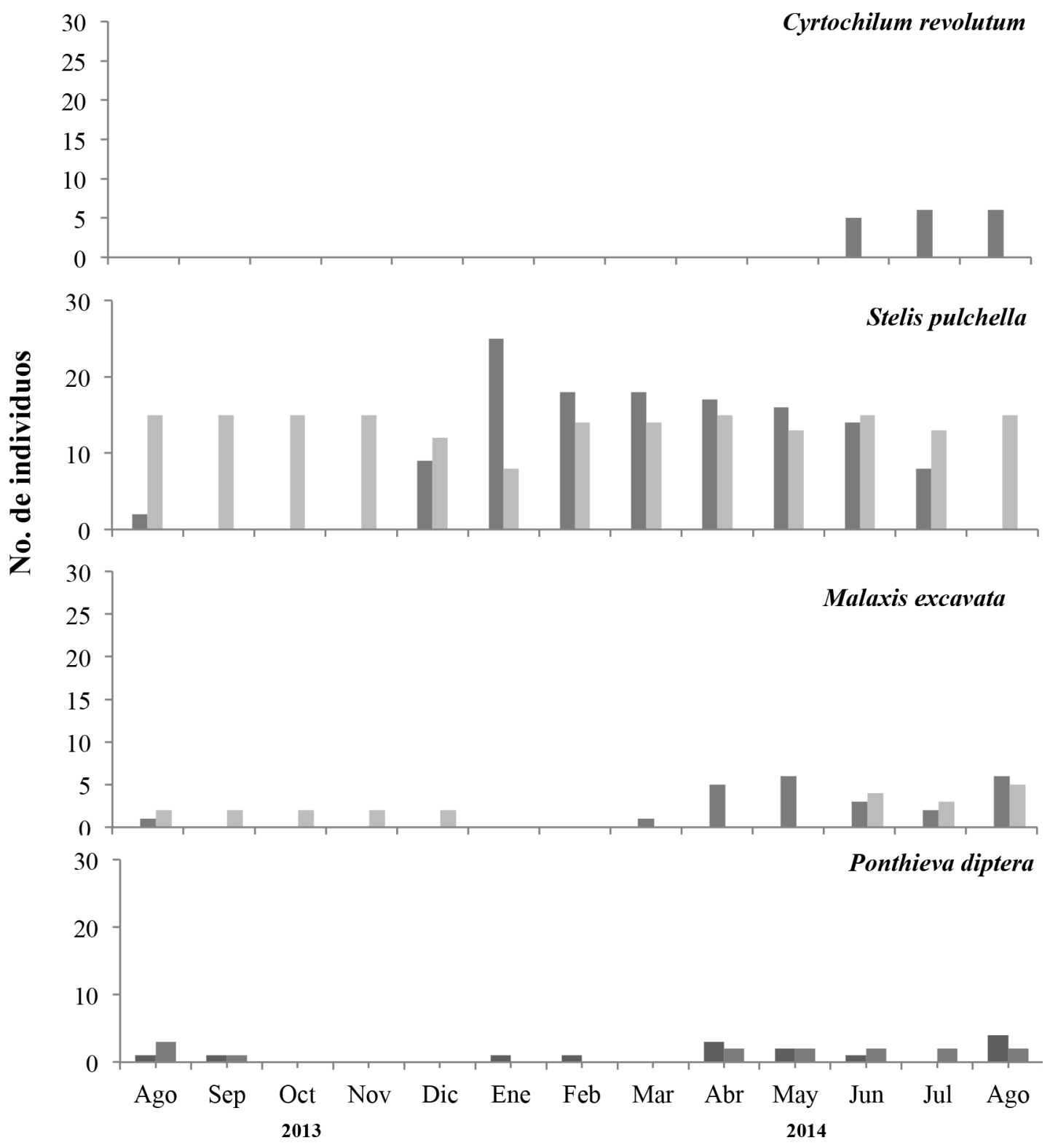

\section{Meses}

FiguRA 2. Número de individuos en estadio fenológico por especie. Barras grises oscuras corresponden a la floración (flores en botón y abiertas), barras grises claras fructificación (frutos maduros e inmaduros).

de correlación por rangos de Spearman $(r)$ para cada especie $\leq 0.05$ (Talora \& Morellato 2000) entre cada variable fenológica monitoreada y los valores mensuales de las variables climáticas del mes inmediato, de uno y de dos meses previos (Sáenz et al. 2003). Las pruebas estadísticas se realizaron con el programa IBM SPSS Statistics 22 (Field 2013).
Resultados. Los resultados en este estudio muestran que las cuatro especies de orquídeas estudiadas presentaron actividad reproductiva entre agosto 2013 y agosto 2014. Respecto a C. revolutum sólo presentó actividad de floración intermedia, mientras que no se encontraron individuos en fruto (Fig. 2; flores en botón, $\mathrm{F}=26.6 \mathrm{P}=0.00$; flores abiertas $\mathrm{F}=26.06 \mathrm{P}=0.00$ ). El 


\section{FLORACIÓN}
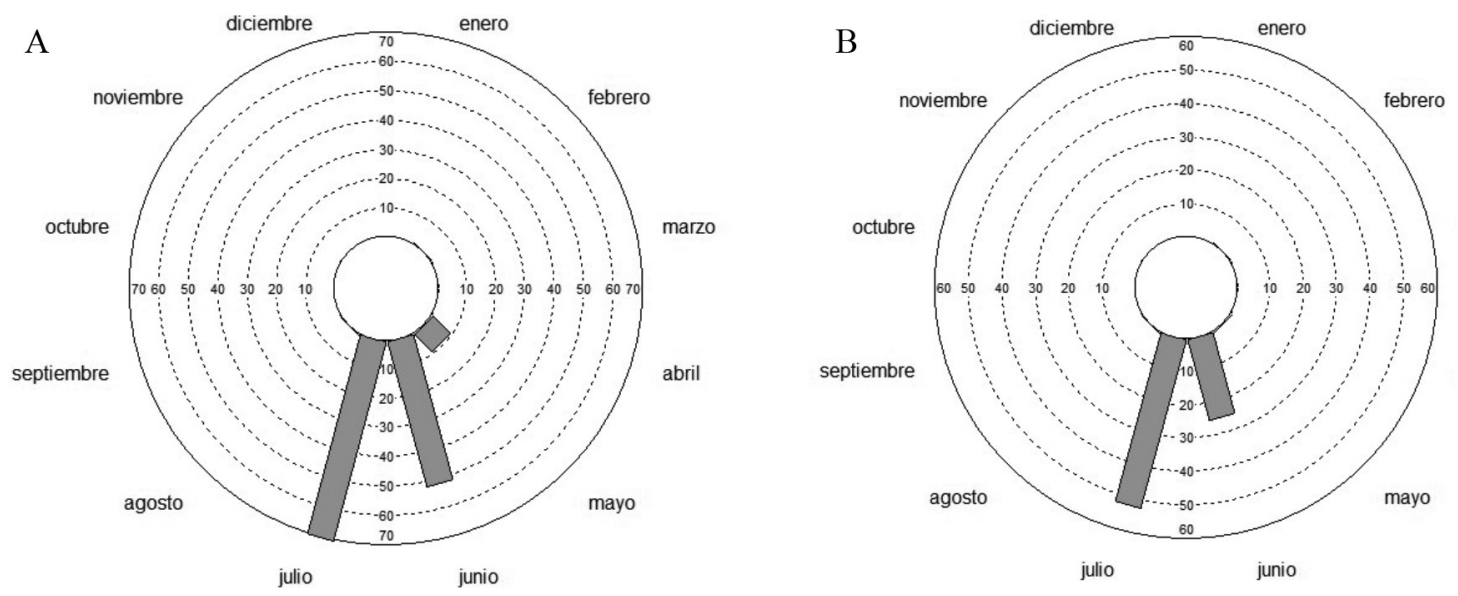

FIgURA 3. Histogramas circulares de la fenología reproductiva (floración-fructificación) de Cyrtochilum revolutum. A, número de flores en botón. $\mathrm{B}$, número de flores abiertas.

pico de floración ocurrió en julio (Fig. 3). Teniendo en cuenta que no se registró fructificación en ninguno de los individuos estudiados.

Para S. pulchella, la mayoría de los individuos florecieron entre diciembre y julio por lo que se consideró que la floración es extendida (Fig. 2). El pico de floración ocurrió en enero tanto para flores en botón $(\mathrm{F}=159.23 \mathrm{P}=0.00)$ como para flores abiertas $(\mathrm{F}=123.16$ $\mathrm{P}=0.00$; Fig. 4). Presentó individuos en fruto durante todo el año, por lo que se consideró como continua. Sin embargo, el pico de producción de frutos verdes ocurrió en agosto $(\mathrm{F}=51.74 \mathrm{P}=0.00)$, mientras que el pico de maduración en diciembre $(\mathrm{F}=72.08 \mathrm{P}=0.00)$.

Se encontró que $M$. excavata aunque tuvo pocos individuos en flor o en fruto durante el año de estudio (Fig. 2) presentó un patrón de floración $\mathrm{y}$ de fructificación extendida (ANOVA de medidas repetidas, floración $\mathrm{F}=45.64 \mathrm{P}=0.000$; fructificación $\mathrm{F}=35.27 \mathrm{P}=0.000$; Fig. 2). Así, la mayor actividad floral ocurrió entre marzo y julio con el mayor número de flores abiertas en abril-mayo (Fig. 5). La fructificación sucedió entre junio y diciembre (Fig. 2) presentándose la mayor producción de frutos verdes en junio-julio mientras que la maduración y liberación de semillas se registró en noviembre-diciembre (Fig. 5).

La especie $P$. diptera también mostró pocos individuos en flor y en fruto durante el año y por ende, no se presentaron picos marcados de actividad fenológica (frutos inmaduros F=23,85 P: 0,02 y frutos maduros F=22,18 P: 0,04; Fig. 2). Por tal razón, no se sugiere ningún patrón de duración de la floración y fructificación. De todas formas el mayor número de flores abiertas se registró para el mes de mayo (Fig. 6), mientras que la liberación de semillas (maduración) se presentó entre julio-septiembre (Fig. 6).

Relación de la fenología con las variables climáticas -. Durante el período de estudio, la temperatura media fue de $16.5^{\circ} \mathrm{C}$, con un máximo de $26.2^{\circ} \mathrm{C}$ en el mes de septiembre (Fig. 7). La humedad relativa osciló entre 78 y $65 \%$ y la precipitación total fue de $943.4 \mathrm{~mm}$, donde enero de 2014 fue el mes más seco con 14.8 $\mathrm{mm}$, y noviembre de 2013 el mes más lluvioso con $201.8 \mathrm{~mm}$. Aunque las temperaturas de septiembre de 2013 y la precipitación de noviembre del mismo año fueron muy altas, el periodo de estudio no coindice con un evento de El Niño ni La Niña. El patrón general se ajusta con los promedios multianuales registrados por el IDEAM para el periodo (1981-2010; IDEAM 2017).

La floración de $C$. revolutum se correlacionó de forma positiva con la precipitación. Así, para el periodo de estudio, la formación de botones florales y flores abiertas ocurrió uno y dos meses después de las mayores precipitaciones. De igual forma, la floración coincide con las menores temperaturas mínimas registradas, por lo cual se presentó una correlación negativa significativa (Tabla 2). 


\section{FLORACIÓN}
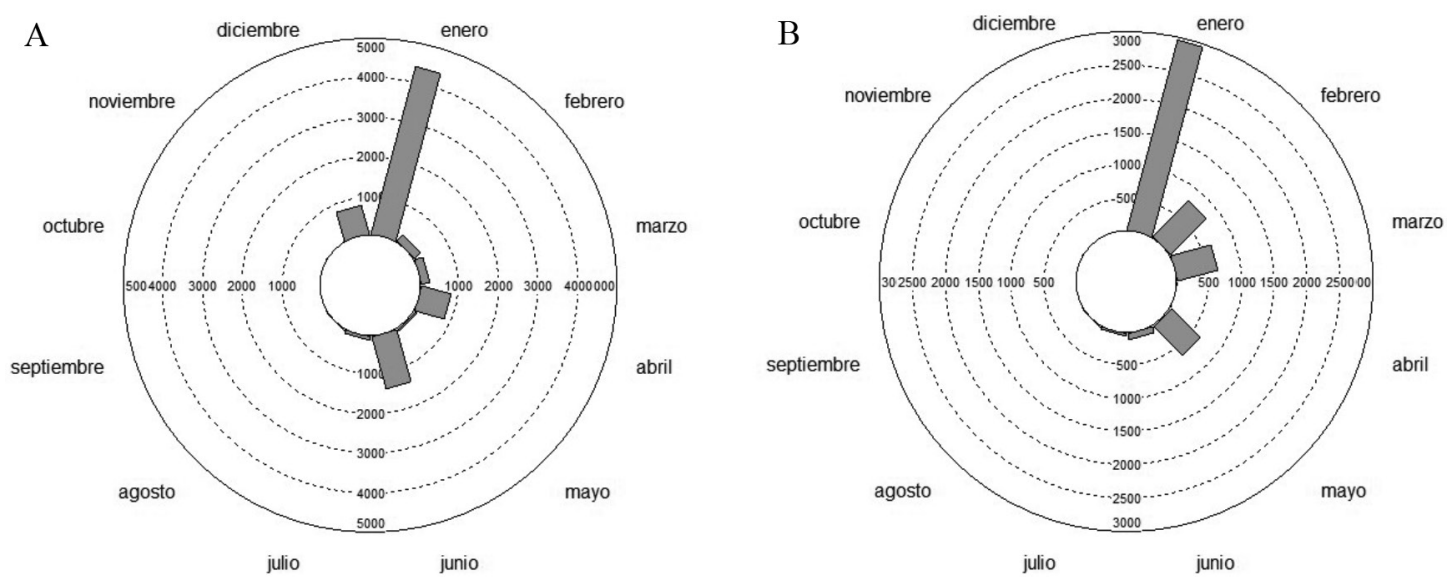

\section{FRUCTIFICACIÓN}
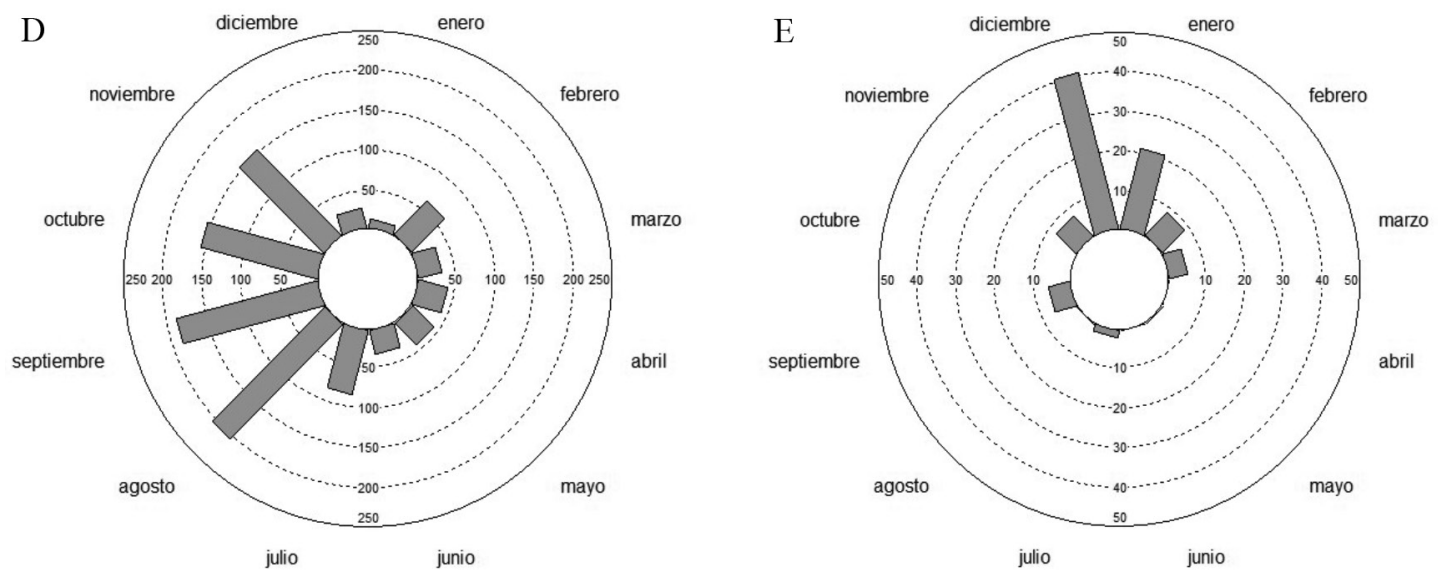

FIgURA 4. Histogramas circulares de la fenología reproductiva (floración-fructificación) de Stelis pulchella. A, número de flores en botón. $\mathrm{B}$, número de flores abiertas. C, número de frutos inmaduros. $\mathrm{D}$, número de frutos maduros.

Para el caso de S. pulchella, se encontró que la floración se presentó en los meses más fríos (de menor temperatura) y con menor humedad relativa del año (Tabla 2). En contraste en los momentos de mayor humedad y mayor temperatura se presentó la producción de frutos verdes. La apertura de las cápsulas ocurrió en los meses más secos del año estudiado (Tabla 2).

La relación entre la fenología de M. excavata y las variables climáticas fue significativamente positiva para la floración y la precipitación (Tabla 2). Así, esta especie floreció durante la primera estación de lluvias entre marzo y mayo. La producción de frutos (inmaduros) ocurrió inmediatamente después de la floración, y se correlacionó con la precipitación de dos meses antes. La cantidad de frutos verdes aumentaron a medida que la humedad relativa aumentó. En contraste, teniendo en cuenta que la dispersión de los frutos es por viento, la maduración y apertura de las cápsulas ocurrió en los meses más secos, con base en la correlación negativa encontrada entre la cantidad de frutos maduros y la precipitación (Tabla 2).

Ponthieva diptera se vio influenciada tanto por la precipitación como por la temperatura. Las lluvias 


\section{FLORACIÓN}
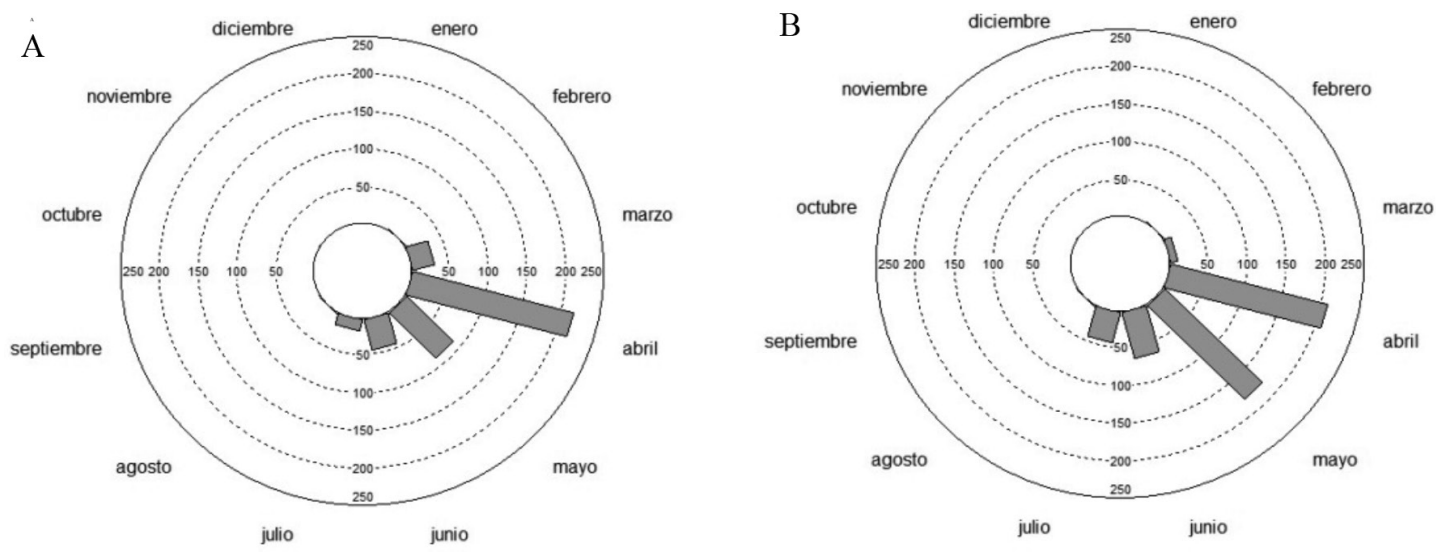

\section{FRUCTIFICACIÓN}
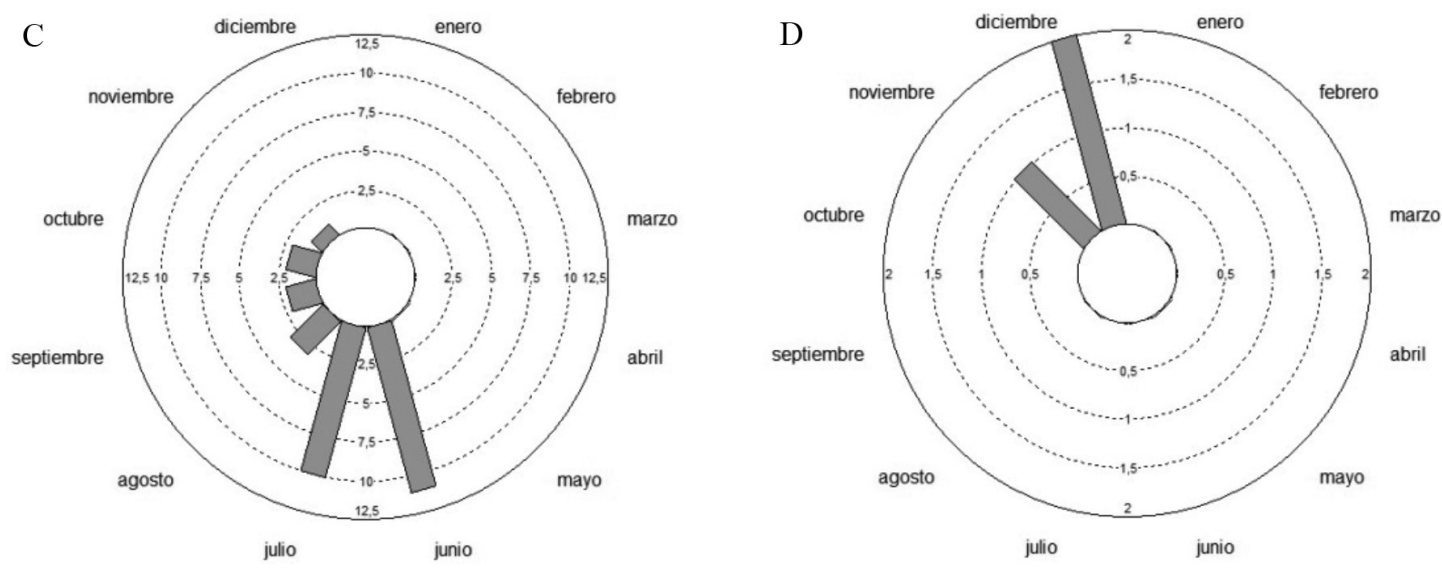

Figura 5. Histogramas circulares de la fenología reproductiva (floración-fructificación) de Malaxis excavata. A, número de

flores en botón. B, número de flores abiertas. C, número de frutos inmaduros. D, número de frutos maduros.

ocurridas durante el mes inmediato se relacionaron con la apertura de las flores en mayo (0.68) así como la producción de frutos verdes ocurrió entre abril y junio (Tabla 2). Se encontró una correlación positiva entre la floración y la temperatura media. La maduración de los frutos ocurrió en los meses de agosto y septiembre meses con la mayor humedad relativa $(\mathrm{rs}=0.76, \mathrm{p}<0.05$; $\mathrm{rs}=0.64)$ al evento (Tabla 2).

Discusión. Los principales estudios en fenología se han realizado para arbustos, árboles, palmas y lianas en regiones templadas y tropicales (e.g. Kotchmer \& Handel
1986, Ibarra et al. 1991, Roldán \& Larrea 2003, Parada et al. 2012, Sánchez-Landaverde 2014), pero en menor frecuencia para herbáceas o epífitas (pero ver Ely-Bali et al. 2010). Los estudios realizados para estos grupos se basan en plantas cultivadas y registros de herbario (e.g. Ospina-Calderón et al. 2007, Sánchez-Landaverde 2014). Así, aunque los registros corresponden sólo a un ciclo anual, este trabajo constituye un aporte al conocimiento in situ de la fenología reproductiva de algunas especies de orquídeas, generando información base para su manejo y futuros planes de conservación.

En términos generales, los resultados de este estudio 


\section{FLORACIÓN}
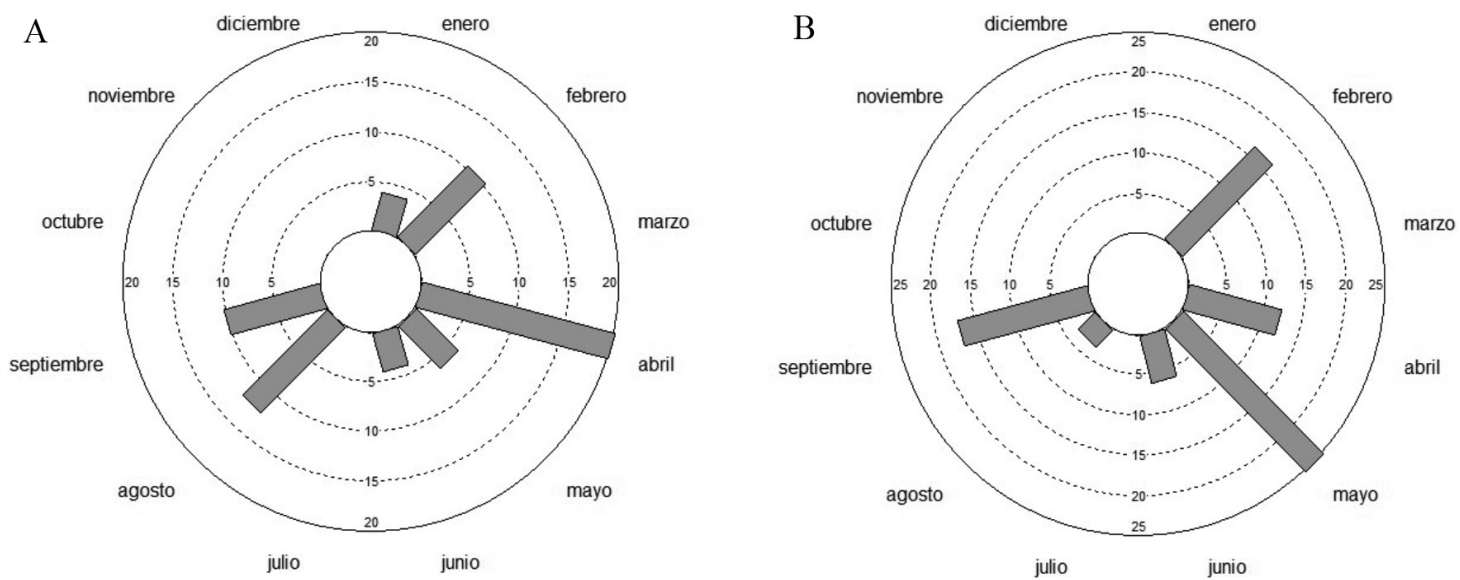

\section{FRUCTIFICACIÓN}
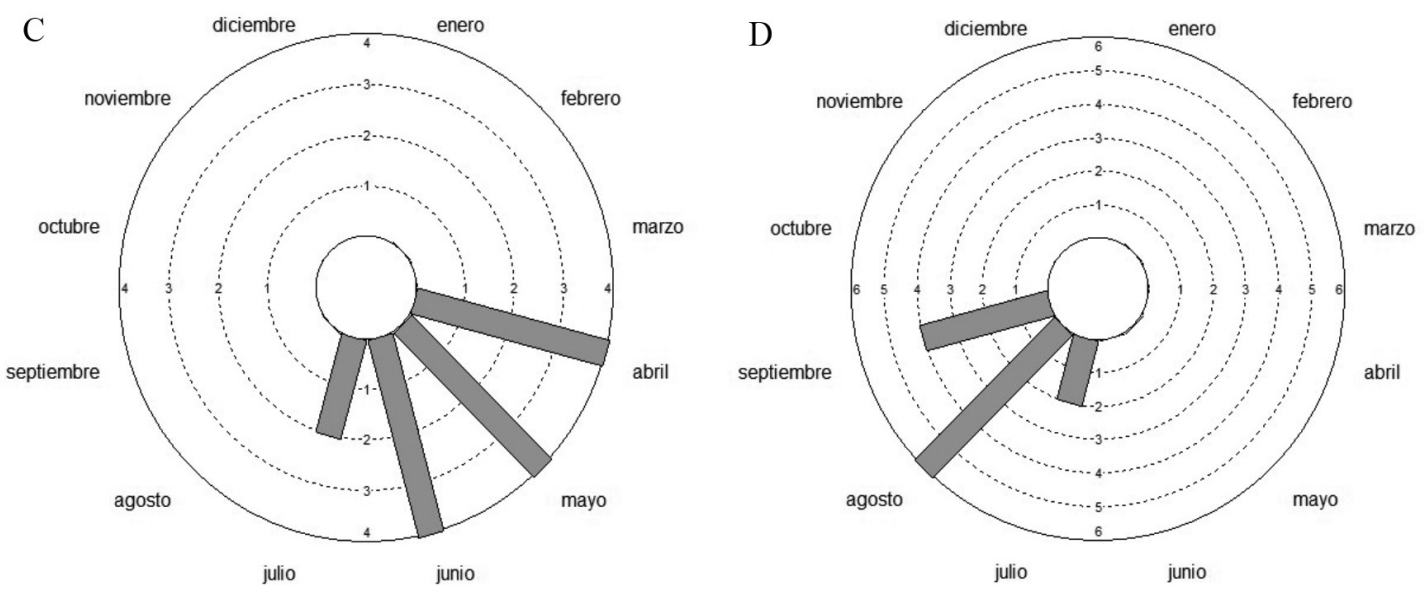

FiguRA 6. Histogramas circulares de la fenología reproductiva (floración-fructificación) de Ponthieva diptera. A, número de

flores en botón. B, número de flores abiertas. C, número de frutos inmaduros. D, número de frutos maduros.

muestran que tres de las cuatro especies estudiadas $(C$. revolutum, $M$. excavata y $P$. diptera) presentan una estacionalidad marcada en sus eventos fenológicos, mientras S. pulchella presentó estacionalidad en la floración más no en la fructificación. La relación entre las variables climáticas y la fenología de las especies estudiadas fue diferencial, pues cada especie mostró correspondencia con por lo menos una variable climática. Sin embargo, fue mucho más clara la relación de la floración con las variables climáticas que con la fructificación. Esto coincide con la literatura previa que ha reportado que la florogénesis en orquídeas epífitas, aunque es sensible a factores genéticos propios de cada especie, los cambios ambientales (estacionalidad), cambios en la temperatura (descensos) y disponibilidad de agua son altamente influyentes (Kochmer \& Handel 1986, Steinfort et al. 2012, Sánchez-Landaverde 2014).

Un hallazgo de gran importancia radica en que para las especies C. revolutum, M. excavata y $P$. diptera florecieron en la época de lluvias con correlaciones positivas significativas (Tabla 2). Esto contrasta con la floración de S. pulchella y con Bonilla et al. (2005) quienes encontraron que la floración en otras especies de orquídeas está más relacionada con la época seca. 

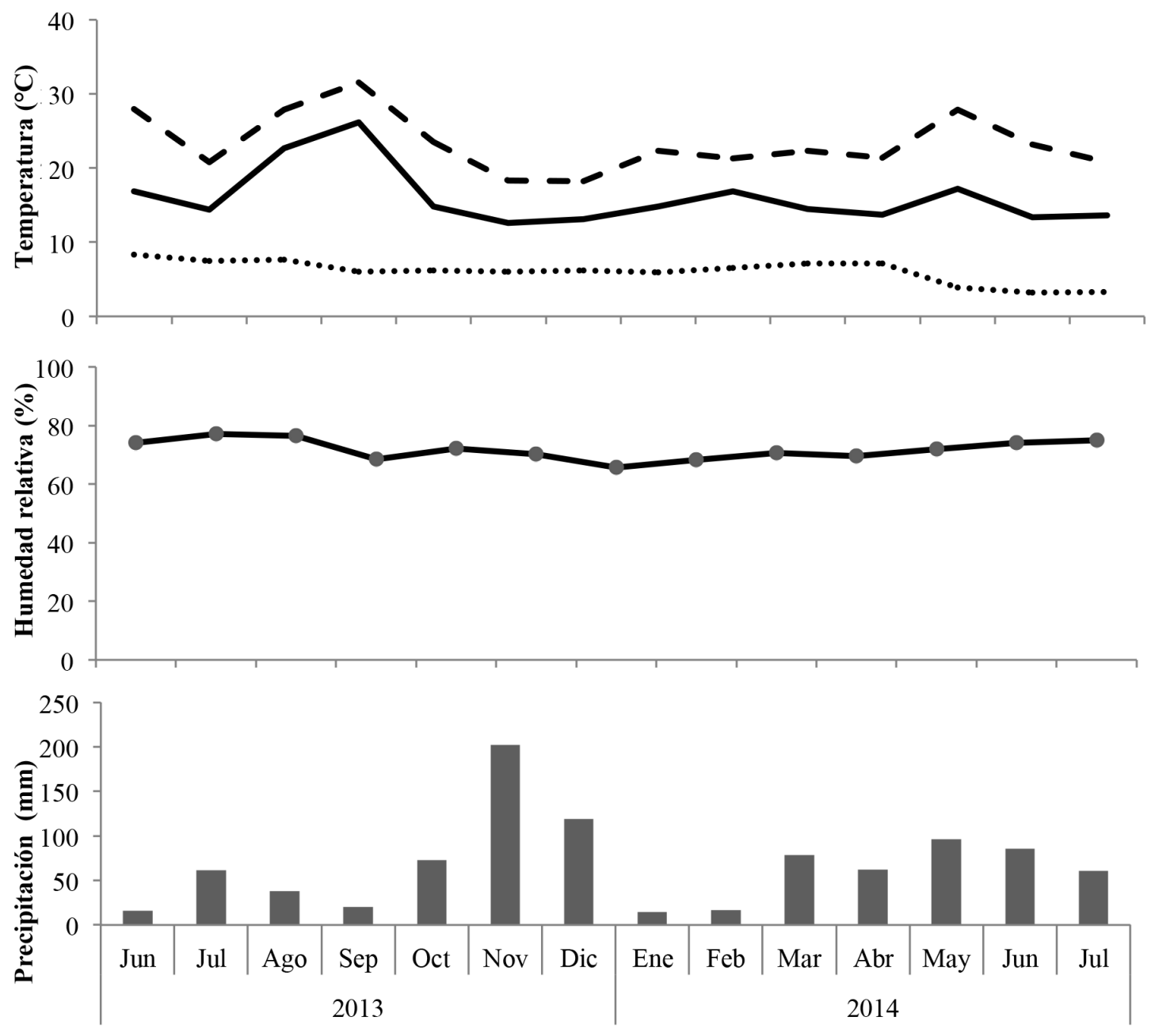

\section{Meses}

FIgURA 7. Variables climáticas registradas en el área de estudio para el periodo agosto de 2013- julio de 2014. Temperatura máxima (línea punteada), media (línea continua) y mínima (punto redondo); Humedad Relativa media mensual y Precipitación total mensual tomadas en las estaciones Santa Cruz de Siecha por la Corporación Autónoma Regional de Cundinamarca y Francisco Wiesner por el Acueducto de Bogotá.

Posiblemente, esta diferencia se debe a que estas tres especies se desarrollan en un medio con una limitada disponibilidad de recursos (suelo con bajo nivel de material orgánica, elevada pendiente y baja humedad en el suelo), que resultan disponibles al aumentar las lluvias, generando condiciones propicias para la floración (van Schaik 1993).

Adicionalmente, en este estudio la fructificación se relacionó de forma significativa con factores ambientales, lo que contrasta con otros estudios en donde esta relación es más débil y se tiende a asociar a otros factores como la fisiología, la disponibilidad de polinizadores y la fecundidad (Roldán \& Larrea 2003, Cuartas-Domínguez \& Medel 2010, SánchezLandaverde 2014, Zárate et al. 2006).

Con relación a $C$. revolutum la variable principal asociada a la floración fue la precipitación, para la que el incremento de las lluvias se asoció con una mayor floración. La ausencia de frutos de esta especie, durante el año de estudio, posiblemente se debe a que 


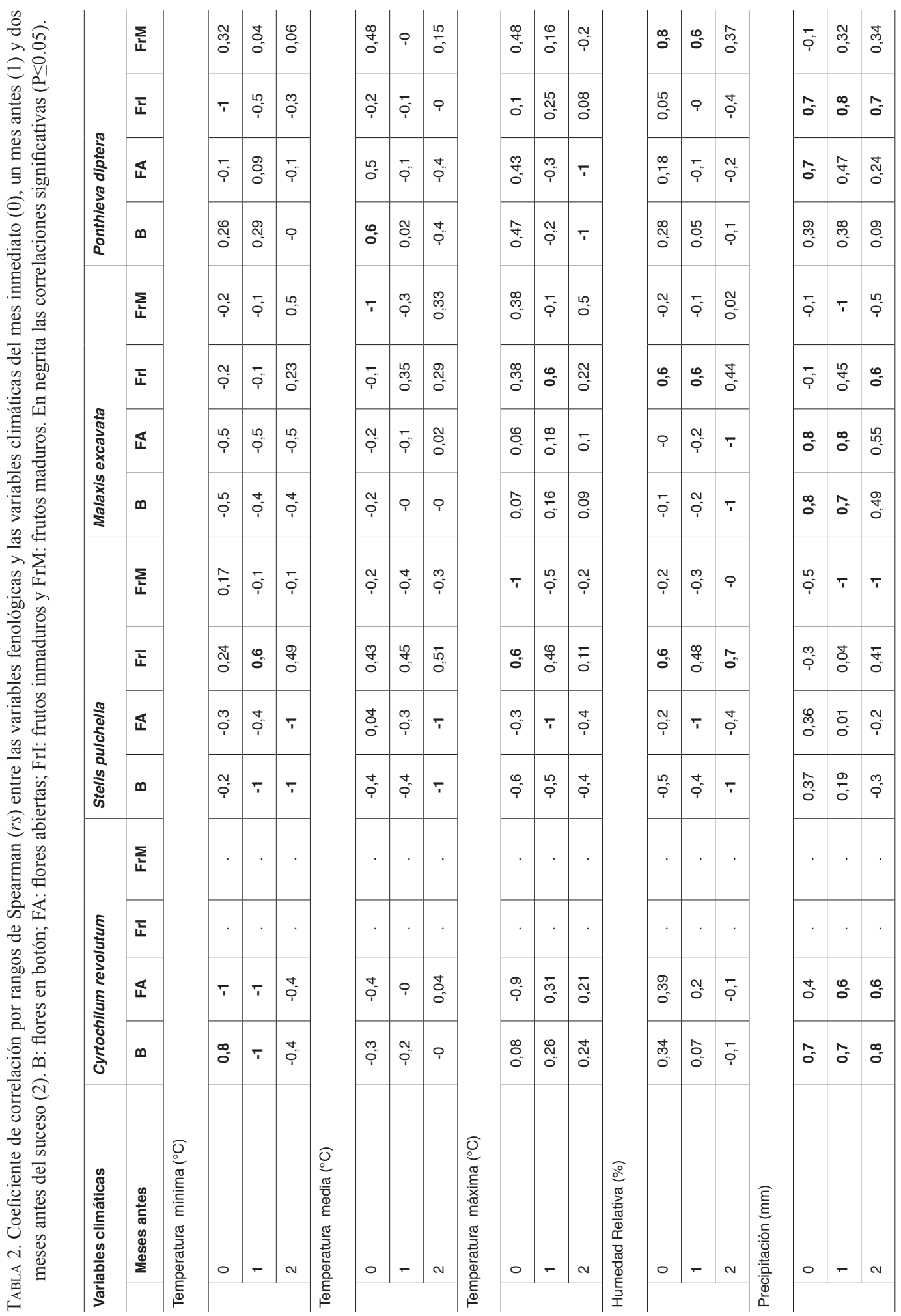


es una planta que desarrolla pseudobulbos grandes y requiere períodos prolongados desde su germinación hasta su madurez. Por lo tanto, se requiere mucho más de un año de seguimiento para poder registrar este fenómeno (Roldán \& Larrea 2003, Cuartas-Domínguez $\&$ Medel 2010). Adicionalmente, teniendo en cuenta que florece en la época húmeda en la que la cantidad de polinizadores, principalmente de la familia Apidae (Torreta et al. 2011) es menor, podría estar afectando la fecundidad de la especie.

En el caso de $S$. pulchella, anteriormente clasificada como Pleurothallis pulchella (Kunth) Lindl., la floración ocurrió al final de la temporada seca y comienzo de las lluvias, períodos donde hay una mayor humedad relativa y temperatura. Esto coincide con lo reportado por Borba \& Semir (2001) en una especie similar, Pleurothallis ochreata, que tiene el pico de floración para la época de mayores precipitaciones. Posiblemente, lo observado en S. pulchella puede deberse a que requiere períodos de estrés hídrico para iniciar la floración (Roldán \& Larrea 2003). Adicionalmente, la relación con la temperatura puede deberse a la intensidad en la fragancia necesaria para atraer polinizadores tal y como ocurre en otras especies de Pleurothallidineas (Borba \& Semir 2001).

La relación entre fructificación y la disminución de la precipitación durante la segunda temporada de lluvias puede explicarse desde la fisiología de la planta y por la disponibilidad de recursos en esta época. Así, similar a lo reportado por Borba y Semir (2001), la lluvia previa se convierte en un importante recurso para la formación de frutos antes de la maduración, que tarda entre tres y cinco meses. Posiblemente, experimentos relacionados con factores limitantes en la producción de frutos pueden aclarar esta tesis.

Por otro lado, la floración de $M$. excavata está altamente relacionada con la cantidad de lluvia, en donde además genera muchas flores de forma secuencial (Aragón \& Ackerman 2001) que se mantienen abiertas por períodos cortos. La presencia de estructuras de almacenamiento como cormos le permite permanecer en estado latente (Sahagún-Godínez 1996) que se rompe con el cambio de estación (Steinfort et al. 2012). De esta forma, se observó que al iniciar las lluvias emergieron tanto los brotes foliares como los florales. En contraste, en la fructificación de M. excavata, la apertura de las cápsulas se relacionó con la época seca, lo cual es congruente con la dispersión por viento de las semillas, que ocurre en las épocas más secas del año.

La floración de $P$. diptera se caracterizó por ofrecer un bajo número de flores de apertura suscesiva durante un largo periodo de tiempo (cuatro meses). Además, esta se relacionó con la temperatura media y con bajas temperaturas máximas durante el periodo de estudio. En el caso de la fructificación la apertura de la única cápsula se relacionó con la mayor humedad relativa; sin embargo, dado a que es un único fruto, es probable que este no sea el real patrón de la especie. Dado el bajo éxito reproductivo (poca cantidad de frutos) habría que hacer más seguimientos en el tiempo para comprender mejor la fenología de esta especie y entender el efecto de los factores endógenos (fisiológicos), nutricionales (disponibilidad de recursos como agua y nutrientes) y disponibilidad de polinizadores (Roldán \& Larrea 2003, Cuartas-Domínguez \& Medel 2010, SánchezLandaverde 2014).

Finalmente, con el fin de aportar al manejo de las orquídeas en estrategias ex situ, se sugiere realizar registros fenológicos continuos por períodos prolongados de más de un año para el mejor entendimiento de su dinámica reproductiva (Ochoa-Gaona et al. 2008), teniendo en cuenta la variabilidad climática entre un año y otro (Kotchmer \& Handel 1986, Ely-Bali et al. 2010). Adicionalmente, aunque el periodo de estudio no correspondió a un evento de El Niño o de La Niña, estos fenómenos pueden afectar de forma considerable la fenología de las especies. A pesar de lo anterior, con base en los resultados encontrados se recomienda, para las especies aquí estudiadas, el empleo del riego y la variación en las temperaturas, si se pretenden reproducir o mantener en condiciones de invernadero (Blanchard et al. 2006).

Agradecimientos. Los autores agradecen al Jardín Botánico de Bogotá José Celestino Mutis por el apoyo financiero a través del programa de estímulos a la investigación Thomas van der Hammen. Al señor Libardo Almeciga por poner a nuestra disposición su predio. A la Universidad Distrital Francisco José de Caldas por su apoyo con las instalaciones y equipos. A las personas que estuvieron apoyando el trabajo de campo. A los Herbarios UDBC, JBB y HUA. Al Biólogo F. Guardiola por su apoyo en los análisis estadísticos. A M. Cuartas, N. Ospina y R. Cortés por sus valiosos comentarios a versiones previas del manuscrito. Dedicamos este trabajo a la memoria del Padre Pedro Ortiz Valdivieso, S.J. $\uparrow$ 


\section{Literatura Citada}

Ackerman, J. (1992). The Orchids of Puerto Rico and the Virgin Islands. First Edition. University of Puerto Rico Press. 147 p.

Alvim, P. T. \& Alvim, R. (1978). Relation of climate to growth periodicity in tropical trees. Cambridge: Cambridge University Press. 464 p.

Aragon, S. \& Ackerman, J. D. (2001). Density effects on the reproductive success and herbivory of Malaxis massonii. Lindleyana, West Palm Beach, 16(1), 3-12.

Ashton, P. S. E. (1988). Dipterocarp biology as a window to the understanding of tropical forest structure. Annual Review of Ecology, Evolution and Systematics, 19, 293-304.

Baker, L. M. \& Baker, O. C. (2006). Orchid species culture, Oncidium/Odontoglossum Alliance. Portland: Timber press. 992 p.

Ballantyne, M. \& Pickering, C. (2012). Ecotourism as a threatening process for wild orchids. Journal of Ecotourism, 11(1), 34-47. Doi: http://dx.doi.org/10.10 80/14724049.2011.628398

Blanchard, M. G. \& Runkle, E. S. (2006). Temperature during the day, but not during the night, controls flowering of Phalaenopsis orchids. Journal of Experimental Botany, 57(15), 4043-4049. Doi: https:// doi.org/10.1093/jxb/erl176

Bonilla, G. M., Chaparro, H. A., Martínez, E. \& Cuervo, M. (2005). Rasgos de historia de vida de las orquídeas clonales terrestres Epidendrum chioneum y Elleanthus ensatus del páramo el granizo. Estrategias adaptativas de plantas del páramo y del bosque altoandino en la Cordillera Oriental de Colombia. Parte V. Universidad Nacional de Colombia. 307-322.

Borba, E. L. \& Semir, J. (2001). Pollinator specificity and convergence in fly-pollinated Pleurothallis (Orchidaceae) species: a multiple population approach. Annals of Botany, 88(1), 75-88. Doi: https://doi. org/10.1006/anbo.2001.1434

Borchert, R., Calle, Z., Strahler, A. H., Baertschi, A., Magill, R. E., Broadhead, J. S., Kamau, J., Njoroge, J. \& Muthuri, C. (2015). Insolation and photoperiodic control of tree development near the equator. New Phytologist, 205(1), 7-13. Doi: 10.1111/nph.12981

Caballero, R. L., Rodríguez, N., Martin, C. (1997). Dinámica de elementos epífitos de un bosque altoandino de la Cordillera Oriental de Colombia. Caldasia, 19(1-2), 311-322.

Chaves, O. M. \& Avalos, G. (2008). Do seasonal changes in light availability influence the inverse leafing phenology of the neotropical dry forest understory shrub Bonellia nervosa (Theophrastaceae). Revista de Biología Tropical, 56(1), 257-268.
Cozzolino, S. \& Widmer, A. (2005). Orchid diversity: An evolutionary consequence of deception? Trends in Ecology \& Evolution, 20(9), 487-494.

Cuartas-Domínguez, M. \& Medel, R. (2010). Pollinatormediated selection and experimental manipulation of the flower phenotype in Chloraea bletioides. Functional Ecology, 24(6), 1219-1227.

Diez, G. M. C. (2002). Biología reproductiva de las plantas de los bosques tropicales. (Trabajo de pregrado, Ingeniería forestal). Medellín: Universidad Nacional de Colombia. $73 \mathrm{p}$.

Dodson, C. H. (2003). Native Ecuadorian Orchids. Volume I-IV. Dodson Trust, Sarasota, Florida. USA.

Dunsterville G. C. K. \& Garay, L. A. (1979). Orchids of Venezuela. Printed for the Botanical Museum of Harvard University. Vol I-III. 1055 p.

Duque-Buitrago, C. A. D., Álzate-Quintero, N. F. \& Otero, J. T. (2014). Nocturnal pollination by Fungus gnats of the Colombian endemic species, Pleurothallis marthae (Orchidaceae: Pleurothallidinae). Lankesteriana, 13(3), 407-417. Doi: http://dx.doi.org/10.15517/lank. v13i3. 14429

Ely-Bali, F., Schwarzkopf Kratzer, T., Rada, R., Fermín, J., León Vargas, Y., \& Gaviria Rincón, J. C. (2010). Respuesta hídrica y fenológica de epífitas y plantas de sotobosque de una selva nublada andina introducidas en un bosque secundario. Ecotrópicos, 23(1), 1-17.

Escobar, R. R. (1994). Native Colombian Orchids: Volume 1-6. Medellín: Editorial Colina.

Field, A. (2013). Discovering statistics using IBM SPSS statistics. Sage. 957 p.

Gentry, A. H. \& Dodson, C. H. (1987). Diversity and biogeography of Neotropical vascular epiphytes. Annals of the Missouri Botanical Garden, 74(2), 205-233. Doi: $10.2307 / 2399395$

Giraldo, G. \& Betancur, J. (2011). Guía de campo de las orquídeas de Santa María (Boyacá, Colombia). Serie guías de campo del Instituto de Ciencias Naturales, Universidad Nacional de Colombia No. 9. Instituto de Ciencias Naturales, Universidad Nacional de Colombia, Bogotá. 188 p.

Guerrero, D. E., Zambrano, W. R., Salazar, A., Lamprea, D. A., Vásquez, B. J., Sandoval, T. J., Posada, A. (2008). Formulación de una cooperativa de servicios turísticos en los municipios de Sopó y La Calera, Cundinamarca (Colombia). Actualidad \& Divulgación Cientifica, 11(1), 175-184.

Hágsater, E. \& Soto, M. (2008). Icones Orchidacearum Fascicle 10, Orchids of México, Part 4. México. 240 p.

Hudson, I. L. \& Keatley, M. R. (Eds.). (2009). Phenological research: methods for environmental and climate 
change analysis. Springer Science \& Business Media $521 \mathrm{p}$.

Ibarra, M. G., Sánchez, G. B. \& González, G. L. (1991). Fenología de lianas y arboles anemócoros en una selva cálido-húmeda de México. Biotropica, 23(3), 242-254.

IDEAM. (2017). Catálogo nacional de estaciones IDEAM Instituto de Hidrología, Meteorología y Estudios Ambientales. Disponible en: http://www.ideam.gov.co/ documents/21021/553571/Promedios+Climatol\%C3 \%B3gicos++1981+-+2010.xlsx/f28d0b07-1208-4a46$8 \mathrm{ccf}-$ bddd $70 \mathrm{fb} 4128$

Kite, G. C. \& Salazar, G. A. (2008). Composición química del olor de la inflorescencia de Malaxis rzedowskiana (Orchidaceae). Revista Mexicana de Biodiversidad, 79(1), 153-157.

Kochmer, J. P. \& Handel, S. N. (1986). Constraints and competition in the evolution of flowering phenology. Ecological Monographs, 303-325.

Lemus-Jiménez, L. J. \& Ramírez, N. (2002). Fenología reproductiva en tres tipos de vegetación de la planicie costera de la península de Paraguaná, Venezuela. Acta Científica Venezuelana, 53(4), 266-278.

Montenegro, A. \& Vargas, O. (2005). Estrategias de regeneración del banco de semillas en una comunidad de bosque altoandino secundario. En: M. A. Bonilla (Ed.), Estrategias adaptativas de plantas de páramo $y$ del bosque alto andino en la Cordillera Oriental de Colombia (pp. 227-246). Bogotá: Editorial Universidad Nacional de Colombia.

Newstrom, L. E., Frankie, G. W. \& Baker, H. G. (1994). A new classification for plant phenology based on flowering patterns in lowland tropical rain forest trees at La Selva, Costa Rica. Biotropica, 26, 141-159.

Ochoa-Gaona, S., Pérez-Hernández, I. \& De Jong, B. H. (2008). Fenología reproductiva de las especies arbóreas del bosque tropical de Tenosique, Tabasco, México. Revista de Biología Tropical, 56(2), 657-673.

Ospina-Calderón, N., Díaz-Granados, M. \& ViverosBedoya, P. (2007). Observaciones de la polinización de Brassia cf. antherotes Rchb.f. (Orchidaceae) en un relicto de selva sub-Andina en la reserva natural la montaña del ocaso. Universitas Scientiarum, 12(1), 83-95.

Otero, J. T. \& Bayman, P. (2009). Germinación simbiótica y asimbiótica en semillas de orquídeas epífitas. Acta Agronómica, 58(4), 270-276.

Parada, Q. M., Alarcón, J. D. \& Rosero, L. L. (2012). Fenología de la floración de especies ornitófilas de estratos bajos en dos hábitats altoandinos del Parque Natural Municipal Ranchería (Paipa-BoyacáColombia). Caldasia, 34(1), 139-154.

Parra, S. E. (2013). Efecto de los patrones del paisaje sobre la diversidad de orquídeas de bosques nublados del
Valle del Cauca (Tesis de maestría: Ciencias Naturales, Biología, Línea Biodiversidad y Conservación). Bogotá: Universidad Nacional de Colombia. 92 p.

Pineda, M. J. (2004). Estructura y composición de las comunidades de orquídeas epífitas (Orchidaceae) en un bosque de niebla con diferentes grados de tala selectiva, en Valle del Sibundoy, Alto Putumayo (Trabajo de pregrado, Biología). Cali: Universidad del Valle. 46 p.

Quiroga, D., Martínez, M. \& Larrea, D. (2010). Pollination systems of five species of orchids growing under greenhouse conditions. Ecología en Bolivia, 45(2), 131-137.

Ramírez, N. (2009). Correlaciones entre la fenología reproductiva de la vegetación y variables climáticas en los altos llanos centrales venezolanos. Acta Botánica Venezuelica, 32(2), 333-362.

Reich, P.B. (1995). Phenology of tropical forests: Patterns, causes and consequences. Canadian Journal of Botany, 72, 164-174.

Roldán, A. I. \& Larrea, D. M. (2003). Fenología de 14 especies arbóreas y zoócoras de un bosque yungueño en Bolivia. Ecología en Bolivia, 38(2), 125-140.

Romero, G. \& Carnevali, G. (2000). Orchids of Venezuela, An illustrated Field Guide. Second Edition. Armitano Editores.

Sáenz, L. C., Gutiérrez, B. \& Alcolado, S. M. (2003). Fenología, agrobiología y producción del Olivar en Almodóvar del campo (Castilla-La Mancha). Anales del Jardín Botánico de Madrid, 60(1), 73-81.

Sahagún-Godínez, E. (1996). Trends in the phenology of flowering in the Orchidaceae of western México. Biotropica, 28(1), 130-136. Doi: 10.2307/2388778

Sánchez-Landaverde, E. (2014). Patrones fenológicos reproductivos y análisis climático de un encinar en Amealco de Bonfil, Querétaro. Santiago de Querétaro: Universidad Autónoma de Queretaro. 64 p.

Sarmiento, J. (2007). La familia Orchidaceae en Colombia. Actualidades Biológicas. IV Congreso Colombiano de Botánica. Medellin-Colombia: Universidad de Antioquia.

Schweinfurth, C. (1958). Orchids of Perú, Part 1-4 Fieldiana, Bot. 30: 1026p. Printed in the USA by Chicago Natural History Museum Press.

Singer, R. (2009). Morfología floral y polinización de orquídeas: el segundo libro de Charles Darwin. Acta biológica Colombiana, 14(1), 337-348.

Steinfort, U., Cisternas, M., García, R., Vogel, H. \& Verdugo, G. (2012). Phenologycal cycle and floral development of Chloraea crispa (Orchidaceae). Ciencia e Investigación Agraria, 39(2), 377-385.

Stevenson, P. R., Castellanos, M. C., Cortés A. I. \& Link, A. (2008). Flowering patterns in a seasonal tropical 
lowland forest in western Amazonia. Biotropica, 40(5), $559-567$.

Talora, D. C., \& Morellato, P. C. (2000). Fenología de espécies arbóreas em floresta de planície litorânea do sudeste do Brasil. Revista brasileira de Botânica, 23(1), 13-26.

Torretta, J. P., Gomiz, N. E., Aliscioni, S. S. \& Bello, M. E. (2011). Biología reproductiva de Gomesa bifolia (Orchidaceae, Cymbidieae, Oncidiinae). Darwiniana, 49(1), 16-24.

Tremblay, R. L., Pomales-Hernández, G. \& MendezCintron, M. (2006). Flower phenology and sexual maturation: Partial protandrous behavior in three species of orchids. Caribbean Journal of Science, 42(1), 75-80.

van Schaik, C. P., Terborgh, J. W., \& Wright, S. J. (1993). The phenology of tropical forests: adaptive significance and consequences for primary consumers. Annual Review of Ecology and Systematics, 24, 353-377.

Vargas, J.O. 1986. Estudios ecológicos en un relicto de bosque de Weinmannia tomentosa y Drimys granadensis en la región de Monserrate. Perez-Arbelaezia, 1(3), 338-356.

Vieira-Faria, M., Andrade, M., Bittencourt, N. \& Carvalho, O. R. (2007). Flowering phenology, nectary structure and breeding system in Corymborkis flava (Spiranthoideae: Tropidieae), a terrestrial orchid from a Neotropical forest. Australian Journal of Botany, 55, 635-642.

Wright, S. J. \& van Schaik, C.P. (1994). Light and the phenology of tropical trees. The American Naturalist, 143, 192-199.

Wright, S. J. (1996). Phenological responses to seasonality in tropical forest plants. In: S.S. Mulkey, R. L. Chazdon \& A. P. Smith (Eds.), Tropical Forest Plant Ecophysiology (pp. 440-460). Springer US.

Zárate, R., Amasifuen, C. \& Flores, M. (2006). Floración y Fructificación de plantas leñosas en bosques de arena blanca y de suelo arcilloso en la Amazonía Peruana. Revista Peruana de Biología, 13(1), 95-102. 
